\title{
Implante de endoprótese aórtica através de acesso totalmente percutâneo
}

\author{
Total percutaneous access for aortic endograft placement \\ Felipe Nasser ${ }^{1}$, Rodrigo Oliveira Pereira da Silva ${ }^{2}$, José Carlos Ingrund ${ }^{2}$, Marcelo Calil Burihan ${ }^{2}$, \\ Francisco César Carnevale ${ }^{3}$, Tiago do Prado Tonial ${ }^{2}$, Raimundo Teixeira de Araújo Júnior ${ }^{2}$, \\ Adnan Neser $^{2}$
}

\section{Resumo}

$\mathrm{O}$ acesso totalmente percutâneo para o implante de endopróteses aórticas, através da preclose technique, é possível mesmo quando utilizadas bainhas introdutoras de elevado perfil. Relatamos sete casos de tratamento endovascular de doenças da aorta, todos realizados de modo percutâneo, e discutimos os principais aspectos desta técnica.

Palavras-chave: Acesso percutâneo, preclose technique, endoprótese aórtica.

\section{Introdução}

Os sistemas de liberação de endopróteses aórticas, utilizados para o tratamento endovascular de aneurismas ou dissecções da aorta, apresentam um perfil elevado, requerendo a exposição cirúrgica da artéria femoral para a introdução dos mesmos com segurança.

Entretanto, em casos selecionados, utilizando dispositivo específico de fechamento arterial mediado por sutura (Perclose Proglide ${ }^{\circledR}$, Abbott Vascular) alguns autores têm obtido êxito no implante percutâneo de endopróteses aórticas, mesmo quando usados sistemas de liberação ou bainhas introdutoras de grande perfil. A técnica, já previamente descrita e nomeada como preclose technique, consiste em uma adaptação do uso do dispositivo hemostático, com introdução do mesmo no início do procedimento, antes da introdução da bainha introdutora ou do próprio sistema de liberação da endoprótese, mantendo os fios de sutura externamente e fixando o nó ao final do procedimento ${ }^{1}$.

\begin{abstract}
Total percutaneous access for aortic endograft delivery, using the preclose technique, is feasible even with the use of large-diameter introducer sheaths. We report seven cases of percutaneous approach in endovascular treatment of aortic diseases and discuss the main technical aspects.
\end{abstract}

Keywords: Percutaneous access, preclose technique, aortic endograft.

No presente estudo, relatamos sete casos de tratamento endovascular de doenças da aorta, sendo cinco intervenções torácicas e duas abdominais, em que o implante da endoprótese aórtica foi realizado de maneira totalmente percutânea, através da técnica preclose.

\section{Casos}

As características dos casos estudados estão descritas na Tabela 1 .

\section{Discussão}

As endopróteses aórticas apresentam perfil elevado, usualmente superior a 18 F, requerendo a dissecção e exposição cirúrgica das artérias femorais para que sejam introduzidas com segurança, sob visão e controle diretos. Embora disponíveis no mercado brasileiro desde 2003, os dispositivos de fechamento arterial mediado por sutura, que foram desenvolvidos pela indústria com a finalidade de eliminar a necessidade de compressão

\footnotetext{
1. Serviço de Cirurgia Vascular, Hospital Santa Marcelina de Itaquera, São Paulo, SP. Serviço de Radiologia Intervencionista, Universidade de São Paulo (USP), São Paulo, SP.

2. Serviço de Cirurgia Vascular, Hospital Santa Marcelina de Itaquera, São Paulo, SP.

3. Serviço de Radiologia Intervencionista, USP, São Paulo, SP.

Não foram declarados conflitos de interesse associados à publicação deste artigo.

Artigo submetido em 25.06.08, aceito em 18.02.09

J Vasc Bras. 2009;8(2):182-185.

Copyright $($ C 2009 by Sociedade Brasileira de Angiologia e de Cirurgia Vascular
} 
Tabela 1 - Casos de implante de endoprótese aórtica através de acesso totalmente percutâneo

\begin{tabular}{|c|c|c|c|c|c|c|}
\hline Paciente & $\begin{array}{l}\text { Data da } \\
\text { intervenção }\end{array}$ & Indicação & $\begin{array}{l}\text { Endoprótese } \\
\text { utilizada }\end{array}$ & $\begin{array}{l}\text { Perfil do sistema } \\
\text { introdutor }\end{array}$ & $\begin{array}{l}\text { Número de percloses } \\
\text { utilizados }\end{array}$ & $\begin{array}{l}\text { Êxito da } \\
\text { intervenção }\end{array}$ \\
\hline 1 & 08/04/2008 & $\begin{array}{l}\text { Dissecção de aorta } \\
\text { torácica }\end{array}$ & $\begin{array}{l}\text { Relay® (Bolton } \\
\text { Medical) }\end{array}$ & $25 \mathrm{~F}$ & 2 na artéria femoral direita & Sim \\
\hline 2 & $17 / 04 / 2008$ & $\begin{array}{l}\text { Aneurisma de } \\
\text { aorta torácica }\end{array}$ & $\begin{array}{l}\text { Valiant }{ }^{\circledR} \\
\text { (Medtronik) }\end{array}$ & $24 \mathrm{~F}$ & 2 na artéria femoral direita & Sim \\
\hline 3 & $18 / 04 / 2008$ & $\begin{array}{l}\text { Aneurisma de } \\
\text { aorta abdominal }\end{array}$ & $\begin{array}{l}\text { Talent }{ }^{\circledR} \\
\text { (Medtronik) }\end{array}$ & $\begin{array}{l}22 \mathrm{~F} \text { na artéria femoral } \\
\text { direita e } 18 \mathrm{~F} \text { na artéria } \\
\text { femoral esquerda }\end{array}$ & $\begin{array}{l}3 \text { na artéria femoral direita } \\
\text { (corpo principal) } \\
2 \text { na artéria femoral } \\
\text { esquerda (contra-lateral) }\end{array}$ & Sim \\
\hline 4 & $10 / 04 / 2008$ & $\begin{array}{l}\text { Dissecção de aorta } \\
\text { torácica }\end{array}$ & TAG® (Gore) & $24 \mathrm{~F}$ & 3 na artéria femoral direita & Sim \\
\hline 5 & $28 / 04 / 2008$ & $\begin{array}{l}\text { Pseudoaneurisma } \\
\text { de aorta torácica }\end{array}$ & $\begin{array}{l}\text { Valiant }{ }^{\circledR} \\
\text { (Medtronik) }\end{array}$ & $22 \mathrm{~F}$ & 2 na artéria femoral direita & Sim \\
\hline 6 & $03 / 06 / 2008$ & $\begin{array}{l}\text { Aneurisma de } \\
\text { aorta torácica }\end{array}$ & TAG® (Gore) & $22 \mathrm{~F}$ & 2 na artéria femoral direita & Sim \\
\hline 7 & $19 / 06 / 2008$ & $\begin{array}{l}\text { Aneurisma } \\
\text { aortoilíaco }\end{array}$ & $\begin{array}{l}\text { Excluder }{ }^{\circledR} \\
\text { (Gore) }\end{array}$ & $18 \mathrm{~F}$ bilateralmente & 2 em cada artéria femoral & Sim \\
\hline
\end{tabular}

manual e de repouso prolongado no leito após a cateterização femoral, são recomendados para o fechamento percutâneo de bainhas introdutoras de no máximo $10 \mathrm{~F}^{2-5}$.

A técnica de fechamento percutâneo da artéria femoral após implante de endoprótese aórtica foi inicialmente descrita em 1999 por Haas ${ }^{1}$, consistindo no uso, não recomendado pelo fabricante, do dispositivo Perclose Prostar XL® de 10 F (Abbott Vascular). Nessa técnica, denominada preclose technique, o dispositivo de fechamento arterial - Perclose - é introduzido antes do implante da endoprótese, e os fios de sutura são mantidos reparados externamente, com o nó sendo apertado ao final do procedimento. Diversos autores, incorporando modificações sutis na técnica descrita por Haas, têm relatado uma taxa de sucesso superior a $90 \%$, dependendo do perfil da bainha introdutora utilizada ${ }^{2-5}$.

Em todos os sete casos relatados (Tabela 1) a técnica utilizada foi essencialmente a mesma. O preparo pré-operatório incluiu rigorosa técnica asséptica e a disponibilidade de instrumental cirúrgico vascular caso fosse necessária uma conversão cirúrgica.

A punção exclusiva da parede anterior da artéria femoral comum com agulha de punção $16 \mathrm{G}$ foi realizada com marcação prévia da cabeça do fêmur sob radioscopia. Punções acima do ligamento inguinal determinam alto ris- co de hemorragia retroperitoneal e falha do procedimento por impossibilidade de compressão adequada ${ }^{2,4}$.

A seguir, uma bainha introdutora $7 \mathrm{~F}$ é implantada na artéria femoral comum e logo retirada, mantendo-se o fio-guia $0,035 \%$. O primeiro Perclose ${ }^{\circledR}$ é introduzido numa angulação de $30^{\circ}$ medialmente. Os fios de polipropileno 3-0 desse dispositivo são mantidos reparados externamente com pinças Kelly® (Figura 1). O fio-guia 0,035” é reintroduzido antes da retirada do primeiro Perclose para permitir a introdução de um segundo Perclose, agora numa angulação de $30^{\circ}$ lateralmente, sendo os fios de polipropileno também mantidos reparados externamente com

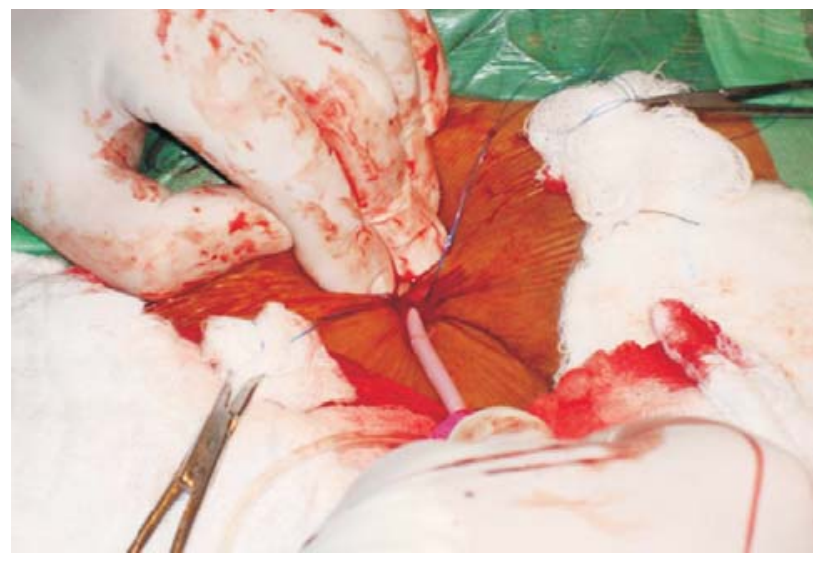

Figura 1 - Manutenção dos fios de sutura reparados externamente no início do procedimento (preclose tecnhique) 
pinças Kelly®. Antes da retirada do segundo Perclose, o fio-guia 0,035 " é reintroduzido e uma bainha $12 \mathrm{~F}$ implantada, com a finalidade de realizar pré-dilatação. Nos pacientes número 3 e número 6 , que foram submetidos ao reparo endovascular de aneurisma de aorta abdominal, todos os passos descritos foram repetidos no outro lado para o implante da extensão contralateral.

Ao término do procedimento, retira-se o sistema de liberação da endoprótese, mantendo-se apenas o fio-guia 0,035 " na artéria femoral. Enquanto o operador executa a compressão manual sobre a artéria femoral proximal, o primeiro auxiliar aperta os dois nós já preparados e mantidos reparados externamente desde o início. Mantém-se a compressão manual por mais 15 minutos. Seguro da adequação da hemostasia, retira-se o fio-guia, mantendo-se a compressão manual por mais 10 minutos. Deve-se enfatizar a importância da manutenção do fio-guia 0,035 " na artéria femoral enquanto os nós do dispositivo são fixados e se comprime manualmente o sítio de acesso. Tal fio-guia, na eventualidade de falha da hemostasia, garante a possibilidade de reintrodução da bainha da endoprótese com a consequente cessação do sangramento e a conversão cirúrgica se necessário ${ }^{3}$.

Alguns autores julgam necessário menor tempo de compressão manual após a retirada das bainhas introdutoras e fixação dos nós do Perclose. Lee et al. ${ }^{2,3}$ por exemplo, recomendam a compressão manual por 5 a 10 minutos e o repouso no leito por 4 a 6 horas. Entretanto, como não revertemos a heparina rotineiramente, mantemos um tempo de compressão manual mais prolongado. De modo geral, o tempo de compressão manual suficiente para adequação da hemostasia costuma ser o mesmo que após a retirada de uma bainha introdutora $5 \mathrm{~F}$ utilizada nos exames diagnósticos. Provavelmente, a manutenção da integridade dos tecidos periarteriais exerça contribuição significativa para a hemostasia do sítio de punção e o êxito da técnica percutânea $^{3}$ (Figura 2).

O procedimento percutâneo apresenta vantagens potenciais que incluem um menor tempo de procedimento, um menor risco de infecção da ferida operatória, além de uma maior satisfação do próprio paciente (Figura 3).

Contudo, o acesso percutâneo com posterior fechamento com dispositivos seladores de punção não é um procedimento isento de riscos. Oclusão arterial aguda com isquemia do membro, trauma arterial, ruptura da sutura

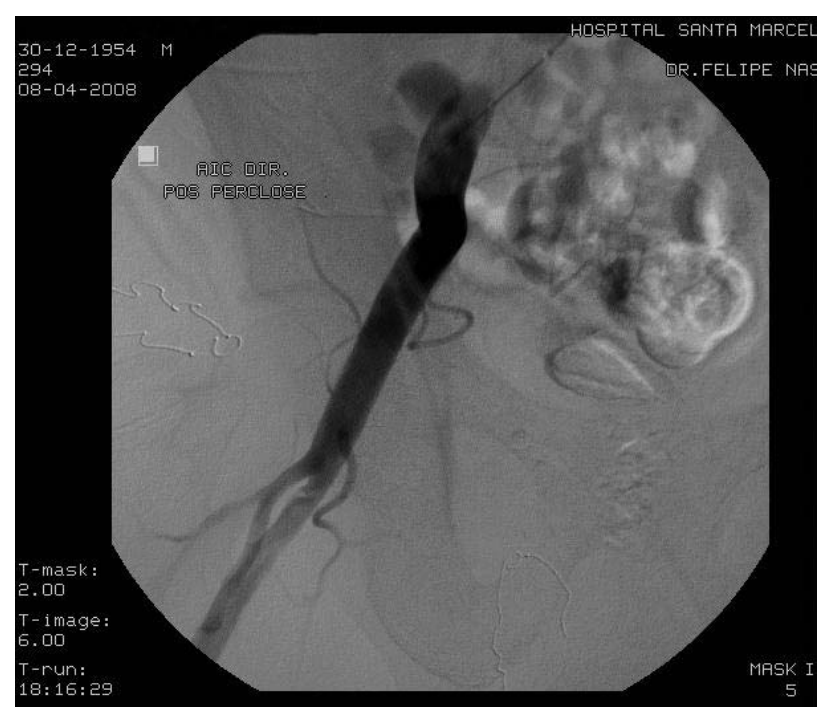

Figura 2 - Arteriografia através de acesso femoral contralateral ao final de nosso primeiro caso (fechamento percutâneo após retirada de bainha $25 \mathrm{~F}$ )

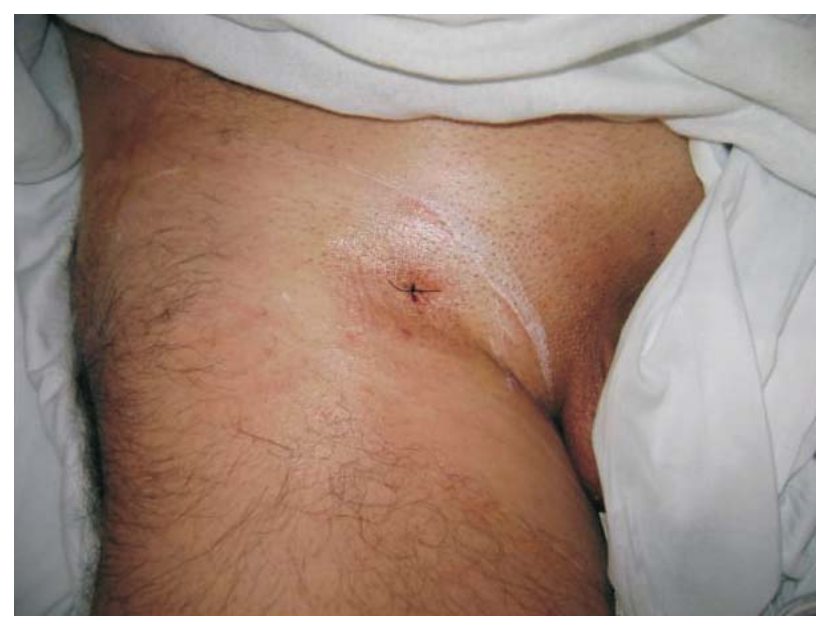

Figura 3 - Aspecto final no primeiro dia pós-operatório após retirada do curativo inguinal; ausência de hematoma ou mesmo equimose

com hemorragia, dissecção arterial, infecção da sutura, formação de pseudoaneurisma, fístula artério-venosa são complicações possíveis da técnica ${ }^{4}$. Em nossa casuística, não houve complicações relacionadas ao fechamento percutâneo, provavelmente devido à adequada seleção dos pacientes.

A adequada seleção dos pacientes para o procedimento percutâneo é de fundamental importância para o sucesso do mesmo. A existência de coagulopatias, a obesidade, a presença de cicatriz inguinal prévia ou calcificação severa do setor ilíaco-femoral configuram contraindicações ao procedimento ${ }^{2-4}$. 
Quanto à técnica anestésica utilizada, é rotina do serviço a realização de raquianestesia em conjunto com a anestesia geral em todos os casos de implante de endoprótese aórtica. Tal conduta visa principalmente a possibilitar a drenagem de líquor, caso necessário, diminuindo a incidência de isquemia medular. Acreditamos que tal abordagem anestésica ofereça também a vantagem de manter o paciente imóvel o tempo necessário para a adequada hemostasia do sítio de punção. Permitindo, inclusive, a conversão cirúrgica com tranquilidade e conforto para a equipe vascular e o paciente se houver falha da técnica percutânea. É importante enfatizar que embora o acesso percutâneo possa parecer especialmente atrativo para o intervencionista sem a formação de cirurgia vascular, o risco de falha é considerável, e uma equipe cirúrgica deve estar à disposição se uma eventual conversão vier a ser necessária.

A experiência com o fechamento percutâneo de endopróteses aórticas vem crescendo em vários centros mundiais, e os resultados quanto à exequibilidade e a segurança do método são entusiasmantes. Lee et al. ${ }^{2,3}$ relatam uma taxa de sucesso global de 94,4\% da técnica preclose, incluindo um índice de sucesso de $92,8 \%$ para bainhas introdutoras de 18 a $24 \mathrm{~F}$. Os mesmos autores obtiveram uma taxa de complicações tardias (avaliação de 6 meses após o procedimento) menor que $2 \%$, confirmando a segurança do método ${ }^{3}$. Apesar do curto tempo de seguimento (máximo de 6 meses após a realização de nosso primeiro caso), não observamos nenhuma complicação relacionada ao acesso percutâneo no seguimento ambulatorial desses pacientes.

$\mathrm{O}$ número limitado de casos percutâneos da nossa experiência se deve à indisponibilidade do dispositivo Perclose na rede pública de saúde, origem da maior parte dos nossos pacientes. O Sistema Único de Saúde não contempla, até o momento, o reembolso de tal material em suas tabelas de procedimentos endovasculares. Entretanto, os benefícios para os pacientes submetidos a essa técnica são óbvios, e, conforme relatamos, a viabilidade e a segurança do método vêm sendo atestadas por diversos trabalhos.

\section{Referências}

1. Haas PC, Kracjer Z, Dietrich EB. Closure of large percutaneous access sites using the Prostar XL percutaneous vascular surgery device. J Endovasc Surg. 1999;6:168-70.

2. Lee WA, Bown MP, Nelson PR, Huber TS. Total percutaneous access for endovascular aortic aneurysm repair ("Preclose" technique). J Vas Surg. 2007; 45:1095-101.

3. Lee WA, Bown MP, Nelson PR, Huber TS, Seeger J. Midterm outcomes of femoral arteries after percutaneous endovascular aortic repair using Preclose technique. J Vas Surg. 2008;47:919-23.

4. Turi ZG. Overview of vascular closure. Endovascular Today. 2008;28-37.

5. Starnes BW, Andersen CA, Ronsivalle JA, Stockmaster NR, Mullenix PS, Statler JD. Totally percutaneous aortic aneurysm repair: experience and prudence. J Vasc Surg. 2006;43:270-6.

Correspondência:

Felipe Nasser

Rua Lincoln Albuquerque 235, 71

CEP 05004-010 - Perdizes, SP

Tel.: (11) 8224.0505

E-mail: nasser.felipe@gmail.com 